Annales Geophysicae (2003) 21: 597-602 (C) European Geosciences Union 2003

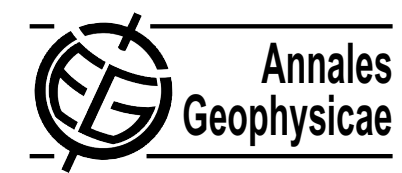

\title{
High-energy charged particle bursts in the near-Earth space as earthquake precursors
}

\author{
S. Yu. Aleksandrin ${ }^{1}$, A. M. Galper ${ }^{1}$, L. A. Grishantzeva ${ }^{1}$, S. V. Koldashov $^{1}$, L. V. Maslennikov ${ }^{1}$, A.M. Murashov ${ }^{1}$, \\ P. Picozza ${ }^{2}$, V. Sgrigna ${ }^{3}$, and S. A. Voronov ${ }^{1}$ \\ ${ }^{1}$ Space Physics Institute, Moscow State Engineering Physics Institute, Kashirskoe shosse 31, 115409 Moscow, Russia \\ ${ }^{2}$ Dept. of Physics, Univ. of Rome "Tor Vergata" and INFN Sez. Rome2, via della Ricerca Scientifica 1, I-00133 Rome, Italy \\ ${ }^{3}$ Dept. of Physics, Univ. of Rome "Roma Tre", via della Vasca Navale, 84, I-00146 Rome, Italy
}

Received: 21 July 2001 - Revised: 21 May 2002 - Accepted: 11 July 2002

\begin{abstract}
The experimental data on high-energy charged particle fluxes, obtained in various near-Earth space experiments (MIR orbital station, METEOR-3, GAMMA and SAMPEX satellites) were processed and analyzed with the goal to search for particle bursts. Particle bursts have been selected in every experiment considered. It was shown that the significant part of high-energy charged particle bursts correlates with seismic activity. Moreover, the particle bursts are observed several hours before strong earthquakes; L-shells of particle bursts and corresponding earthquakes are practically the same. Some features of a seismo-magnetosphere connection model, based on the interaction of electromagnetic emission of seismic origin and radiation belt particles, were considered.
\end{abstract}

Key words. Ionospheric physics (energetic particles, trapped; energetic particles, precipitating; magnetosphereionosphere interactions)

\section{Introduction}

Correlation between short-term variations (bursts) of highenergy charged particle fluxes in near-Earth space and seismic activity was pointed out for the first time at the end of the 1980s (Voronov et al., 1987; Voronov et al., 1989). This conclusion was made on the basis of results, which were obtained from the MARIA experiment on board the SALYUT7 orbital station. Then, the detailed study of electron and proton flux variations below the radiation belt was continued by MARIA- 2 magnetic spectrometer on board the MIR station, ELECTRON instruments on board INTERCOSMOSBULGARIA-1300 and METEOR-3 satellites (Galper et al., 1989; Voronov et al., 1990). The results of these experiments confirmed the existence of correlation between shortterm sharp increases of particle fluxes and seismic processes. Moreover, it was found that the particle flux variations ap-

Correspondence to: S. V. Koldashov

(koldashov@space.mephi.ru) peared several hours before the main shock of strong earthquakes (Aleshina et al., 1992; Galper et al., 1995). Thus, the short-term earthquake precursors in the near-Earth space were experimentally observed.

Using the analysis of spatial distributions of particle bursts and earthquakes with magnitude exceeding 4 (Richter scale), it was also shown that the forthcoming earthquake and its precursor (particle burst) are located on nearly the same Lshells.

Later, two experimental works dedicated to the analysis of the experimental data obtained on board METEOR-3A and OREOL-3 satellites were published (Galperin et al., 1992; Pustovetov and Malyshev, 1993). Authors searched for the seismo-magnetosphere correlation (particle bursts and seismic activity) and confirmed the existence of the phenomenon mentioned above.

The explanation of this phenomenon is based on the local disturbance of radiation belt particle flux caused by ultra low frequency (ULF) electromagnetic emission (EME) of seismic origin (Aleshina et al., 1992; Galper et al., 1995).

It was shown in direct ground-based experiments that this EME can be generated in the earthquake zone several hours before the main shock (Fraser-Smith et al., 1990) and can propagate upwards through the ionosphere into the magnetosphere (Molchanov and Majaeva, 1994). At a certain altitude, the EME can be captured in the geomagnetic field tube (Molchanov et al., 1992) and then propagates as Alfvén wave along the geomagnetic field lines. Reaching the lower radiation belt boundary, the Alvén wave begins to interact with trapped particles, causing particle precipitations as a result of pitch-angle redistribution.

These precipitated particles drift around the Earth along the L-shell, corresponding to the earthquake epicenter location (Galper et al., 1997). This process creates a wave of precipitated particles. Such a wave can make one or more revolutions around the Earth before being damped (Galper et al., 1995; Galper et al., 2000). The instrumentation on board a satellite can observe particle waves as particle bursts, when satellite crosses this disturbed L-shell. 


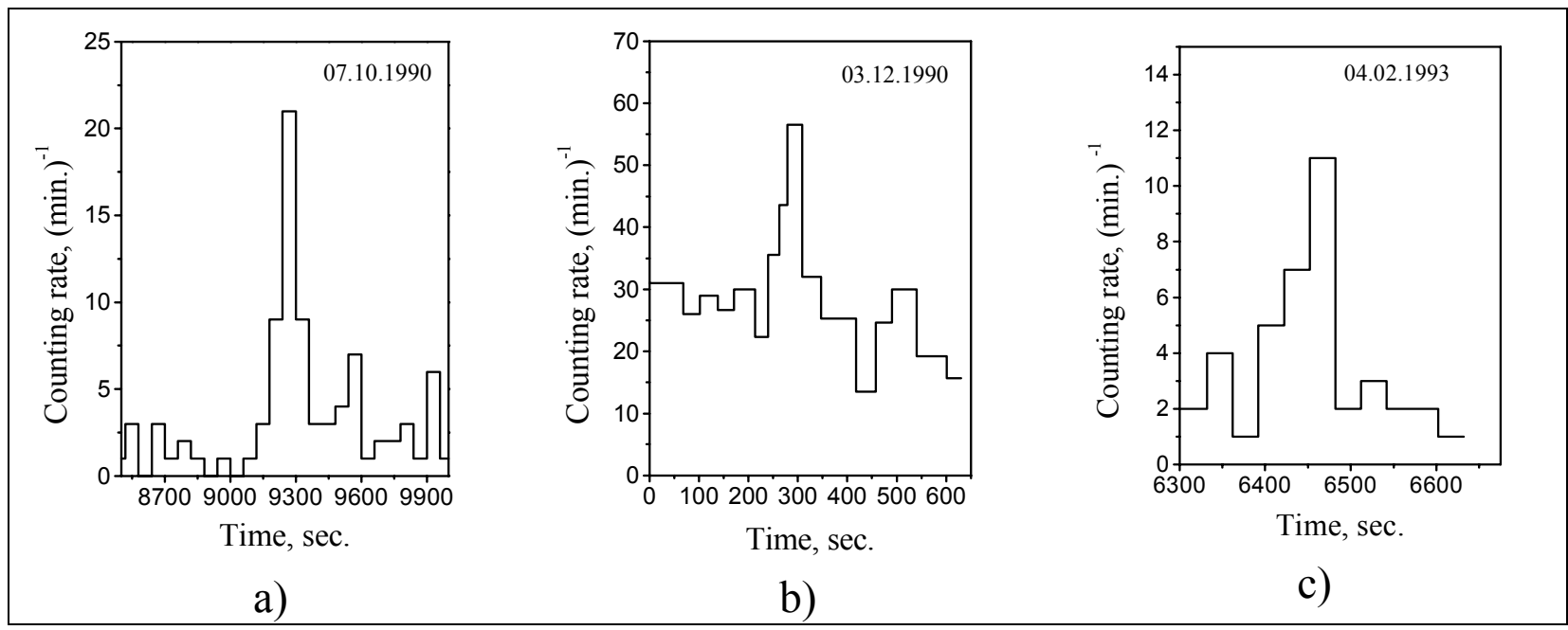

Fig. 1. Examples of electron counting rates along the orbit recorded in MARIA-2 (a), GAMMA-1 (b) and SAMPEX/PET (c) experiments during observation of the particle bursts.

It should be stressed that due to the drift around the Earth, the particle burst of seismic origin can be observed not only over the epicenter but also at any longitude, where satellite crosses the disturbed L-shell.

Here we present the results of the analysis of new experimental data on bursts of high-energy charged particles, obtained by processing of GAMMA-1 and PET experiments and additional information from MARIA-2 and ELECTRON experiments.

\section{Instrumentation}

A brief description of instruments, used to search for the seismo-magnetosphere correlation, is presented below.

The MARIA and MARIA-2 instruments are magnetic time-of-flight scintillator spectrometers (Voronov et al., 1991) that were installed on board the SALYUT-7 and MIR orbital stations $\left(51^{\circ}\right.$ inclination, $400 \mathrm{~km}$ altitude). These instruments provided for detection and identification of protons, electrons and positrons in the energy range 20$200 \mathrm{MeV}$. Measurements were carried out periodically from 1985 to 2000.

The ELECTRON instrument (Galper et al., 1983) was installed on board the METEOR-3 satellite $\left(82^{\circ}\right.$ inclination, $1250 \mathrm{~km}$ altitude). Using a stack of scintillator detectors and a Cherenkov counter, it detected electrons with energies, exceeding $30 \mathrm{MeV}$. The experiment was operational in the period 1985-1986.

Gamma-ray telescope GAMMA-1 (Akimov et al., 1988) was installed on board the astrophysical station GAMMA ( $51^{\circ}$ inclination, $350 \mathrm{~km}$ altitude). In addition to the main scientific information on gamma rays, the instrument recorded charged particle counting rates, in particular, the counting rate of high-energy electrons (energy $>50 \mathrm{MeV}$ ).
In order to select electrons, the scintillator counters, timeof-flight system and gas Cherenkov detector were used in combination with a lead scintillator calorimeter. The measurements were carried out in orbit during 1990-1992.

The SAMPEX (Baker et al., 1993) satellite was launched into $82^{\circ}$ inclination, $600 \mathrm{~km}$ altitude orbit in 1992 . PET instrumentation on board the SAMPEX consisted of a stack of lithium-drifted-silicon detectors which measured proton and electron fluxes. Experimental data (1993-1994) of PET electron channel (4-15 MeV) were used for search of seismomagnetosphere correlation.

\section{Data processing and analysis}

In order to select charged particle bursts we used the counting rate information of the instruments mentioned above. This information was obtained processing measurement data for the MARIA-2 and GAMMA-1 experiments. Information on counting rates of the PET instrument (SAMPEX Level 2 Data) was been received from National Space Science Data Center. For ELECTRON experiment we used particle burst data obtained earlier (Galper et al., 1989).

Sites of spacecraft orbits, which correspond to regions of the near-Earth space with L-shell less than 2 (main part of earthquakes located in the near equatorial region), were selected for the analysis. Moreover, to decrease the background flux and to increase the reliability of particle burst selection, the South Atlantic Anomaly region was excluded from consideration.

Sharp, short-term increases of particle counting rates from tens of seconds to a few minutes were selected as particle bursts if the current counting rate exceeded 4 standard deviations from average value of the background one. Figure 1 


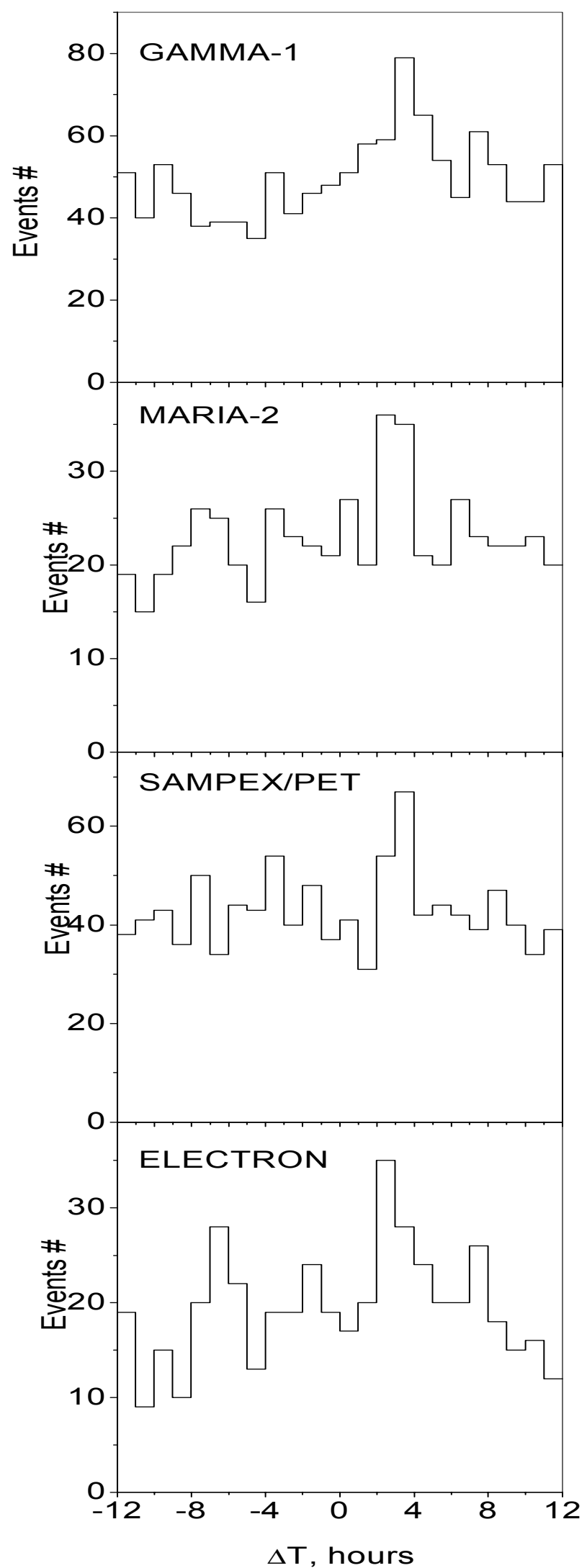

Fig. 2. $\Delta T$ distribution histograms for particle bursts and earthquakes obtained in GAMMA-1, MARIA-2, PET and ELECTRON experiments $(M>4,|\Delta L|<0.1)$. shows typical examples of particle burst observation with on board instruments.

\section{Temporal correlation}

An analysis of temporal correlation between particle bursts and strong earthquakes was carried out using the same procedure for all experiments. Strong earthquakes with magnitudes $M>4$ (Richter scale) from the CNSS Catalog (Malone et al., 1996) were selected in the time interval $\pm 12 \mathrm{~h}$ around the time of particle burst observation. The time difference $(\Delta T)$ between the earthquake and the particle burst was calculated for every earthquake in this temporal range.

$\Delta T=T_{E Q}-T_{P B}$

where $T_{E Q}$ and $T_{P B}$ are the times of occurrence of earthquake and particle burst, respectively.

We then applied this procedure for all observed particle bursts and, after the processing all the events, the histograms of $\Delta T$ distributions were plotted for every experiment. As an additional parameter (its value could be varied) we used the difference between L-shells of the earthquake and the particle burst $(\Delta L)$.

$\Delta L=L_{E Q}-L_{P B}$

where $L_{E Q}$ and $L_{P B}$ are the L-shells of earthquake and particle burst, respectively.

As $L$ coordinate of earthquake, we took the $L$ coordinate of the point at a certain altitude above the epicenter. This altitude coincides with the altitude of the region from which the EME of seismic origin is captured in a geomagnetic field tube and propagates in the magnetosphere along field lines. Such a determination of the $L$ coordinate of an earthquake corresponds to the physical model described above (Aleshina et al., 1992). The altitude of EME capture, as it was estimated by Molchanov et al. (1992), is about $300 \mathrm{~km}$.

Using $\Delta L$ cut (Eq. 2) we could only take into account earthquakes which locate closely to the particle burst in Lspace (values used were $|\Delta L|<0.2,0.1,0.05$ ). It should be emphasized that this cut is in accordance with the physical model of the phenomenon, because the development of local disturbance of the radiation belt caused by the earthquake occurs on an L-shell corresponding to the earthquake.

The $\Delta T$ distribution histograms, obtained using experimental data of MARIA-2, ELECTRON, GAMMA-1 and PET, are shown in Fig. 2.

One can see that the shapes of all 4 histograms are practically the same; notably the peak in the +2 to +5 -h range. The positive shift of this peak in $\Delta T$ histograms means that the particle bursts are observed as short-term earthquake precursors.

Analysis showed that the shape of $\Delta T$ distribution histograms can change significantly under varying values of $\Delta L$ and $M$. Thus, for example, the plot has a very high peak if $M>5$ and $|\Delta L|<0.07$ (Fig. 3), otherwise, the plot is 


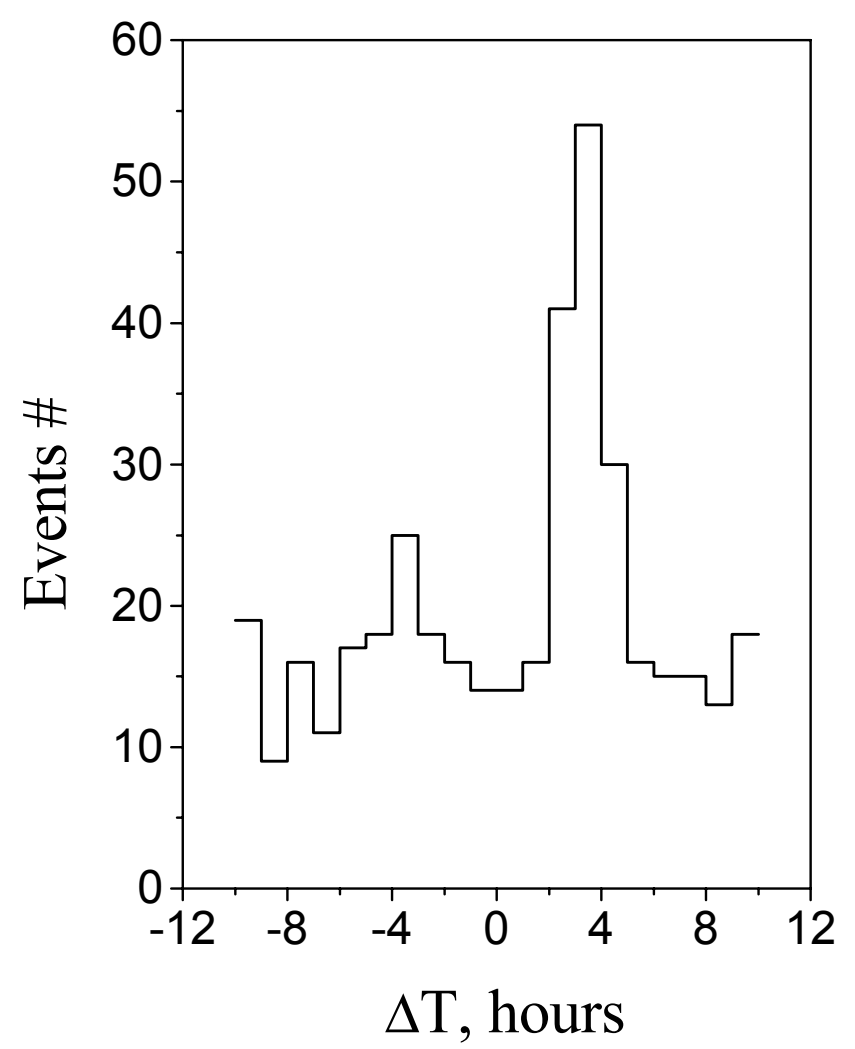

Fig. 3. $\Delta T$ distribution histogram for particle bursts and earthquakes (SAMPEX/PET, $M>5,|\Delta L|<0.07$ ).

practically uniform (without any peaks) if $|\Delta L|>0.5$. Figure 3 presents the plot for PET data. Similar results have been also obtained with data of the other experiments but, unfortunately, statistics accumulated on particle burst observation in these experiments was not enough high for construction of a similar detailed plot. It should be noted that putting the cut on value $|\Delta L|>0.5$ means that only earthquakes for which the L-shells significantly differed from L-shells of particle bursts were taken for analysis. The uniform $\Delta T$ distributions were also obtained if years of experiments and earthquakes were randomly mixed.

\section{Spatial correlation}

In order to estimate the influence of the $\Delta L$ cut on the value of the peak $(2-5 \mathrm{~h})$ in $\Delta T$ distribution histograms (from the point of view of peak evidence), we considered $\Delta L$ as a free parameter and recalculated $\Delta T$ histograms for different values of $\Delta L$. As a quantitative criterion of peak evidence it is convenient to use the value of peak confidence level evaluated in units of standard deviation.

$n \sigma=\left(N_{\mathrm{MAX}}-N_{B G} / \sigma\right)$

Where $n \sigma$ is the confidence level of the peak, $N_{\text {MAX }}$ is the number of events in the peak of $\Delta T$ histogram, $N_{B G}$ is the average number of events in the $\Delta T$ histogram and $\sigma$ is standard deviation from the background value. It is obvious that the confidence level of the peak in $\Delta T$ histograms will change with variation of the $\Delta L$ value.

The confidence level of the peak in histograms of $\Delta T$ versus $|\Delta L|$ is presented in Fig. 4 for the GAMMA-1 and SAMPEX/PET experiments (fitting curves are shown in this figure). We present here plots for only the GAMMA-1 and SAMPEX/PET experiments because the statistics of particle bursts observed in the MARIA-2 and ELECTRON experiments were not enough high for such analysa. The highest values of confidence level (5.5 and 6 standard deviations) are obtained under $\Delta L=0.07$ for both experiments $(|\Delta L|=0.07$ corresponds to the distance about $200 \mathrm{~km}$ in latitude on the Earth's surface for $L=1.4$ ).

A decrease of the confidence level of the peak in the $\Delta T$ histogram due to too small or too large $|\Delta L|$ values, can be explained as follows. Decreasing $|\Delta L|$ to too small a value results in the reduction of event statistics and, hence, the confidence level of the peak falls. On the other hand, applying too large $|\Delta L|$ values takes into account earthquakes that are far away from the particle burst L-shell and so we increase the portion of background events in the total statistics. In this case the peak confidence level also decreases.

\section{Discussion}

Different local disturbances of the radiation belt can cause particle precipitations with a subsequent formation of waves of high-energy charged particles. Such waves can propagate around the Earth along the drift L-shells. If spacecraft crosses this disturbed L-shell, then on board instrumentation observes the particle wave as a particle burst.

One of the reasons for the radiation belt disturbance is the EME of seismic origin which is generated during the earthquake preparation phase (Aleshina et al., 1992; Molchanov and Majaeva, 1994).

The existence of an obvious peak in the $\Delta T$ histograms points out unambiguously that the significant part of particle bursts is connected with seismo-magnetospheric processes.

It is possible to use the $\Delta T$ distribution histogram with a clear peak as an indicator of the presence of seismomagnetospheric correlation. Such an approach allows us to analyze some features of the physical mechanism of this correlation. According to the physical model for this phenomenon (EME of seismic origin which disturbs the radiation belt and results in particle precipitations) the particle burst, as a final result of the precipitation, and the EME should have the same L-shell. The altitude, where EME is captured in a geomagnetic field tube and then propagates along field lines, can be estimated from experimental data on particle burst observations. By considering the capture altitude as a free parameter in the calculations of $\Delta T$ histograms one can obtain the possible range of altitudes of EME capture. It should be noted, that the change of assumed value for the altitude of EME capture gives rise to varying $L$ coordinates of the earthquakes; hence, the relation between real 


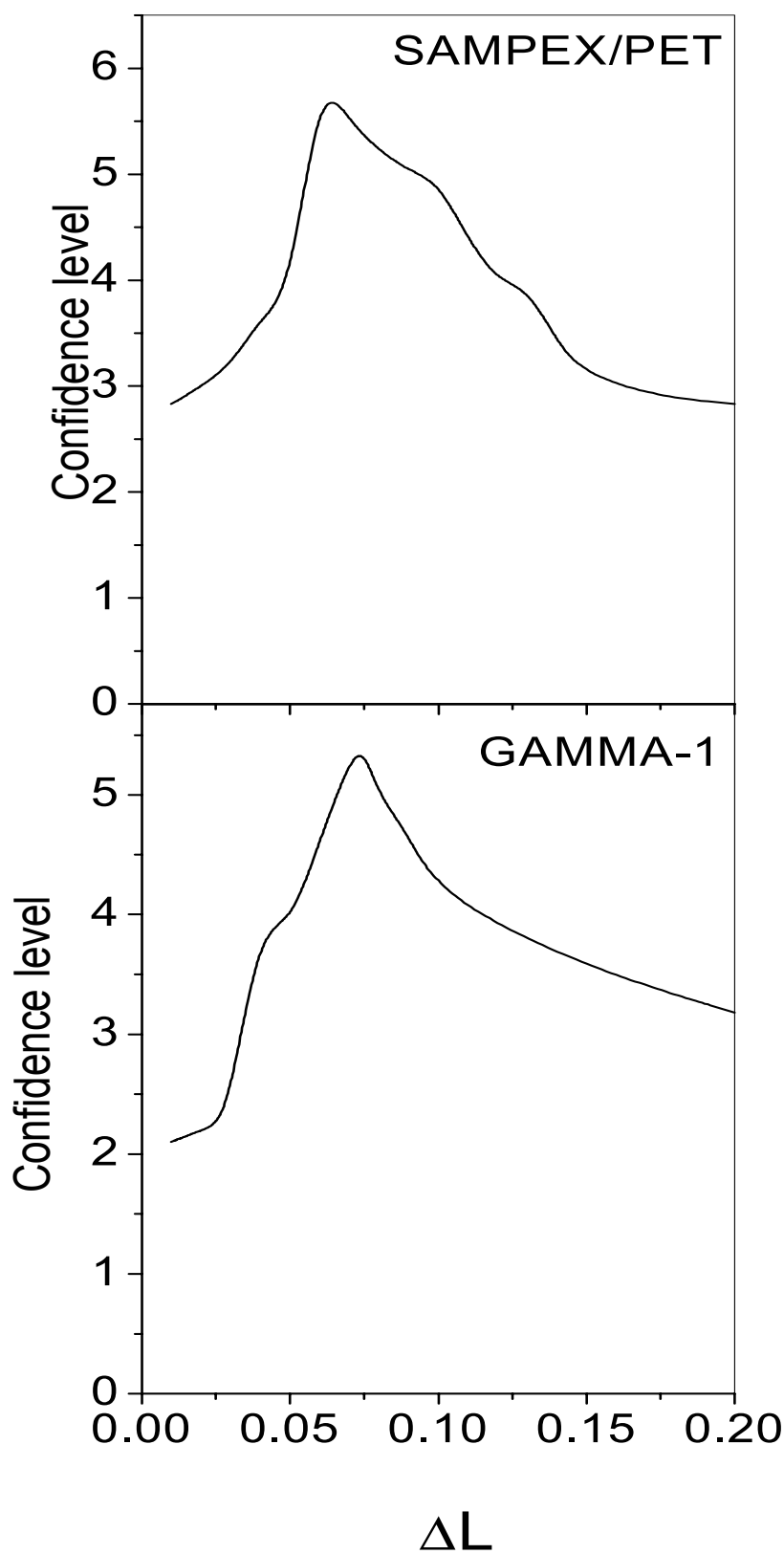

Fig. 4. Confidence level (in number of standard deviations) of the peak in the $\Delta T$ distribution histograms vs. $\Delta L$ (SAMPEX/PET and GAMMA-1).

correlated events (particle bursts and earthquakes) and background events will be also changed in the total statistics.

In Fig. 5 the confidence level ( $n \sigma$, see Eq. 3 ) of the peak in $\Delta T$ distribution histograms is plotted versus assumed values of EME capture altitude for the MARIA-2, GAMMA-1 and SAMPEX/PET experiments (fitting curves are shown in this figure). ELECTRON data is not presented here because of insufficient statistics of particle bursts observed in these experiments for such analyses.

The existence of a maximum in this figure for all experiments confirms the present physical concept of seismomagnetospheric correlation. The capture altitude range of

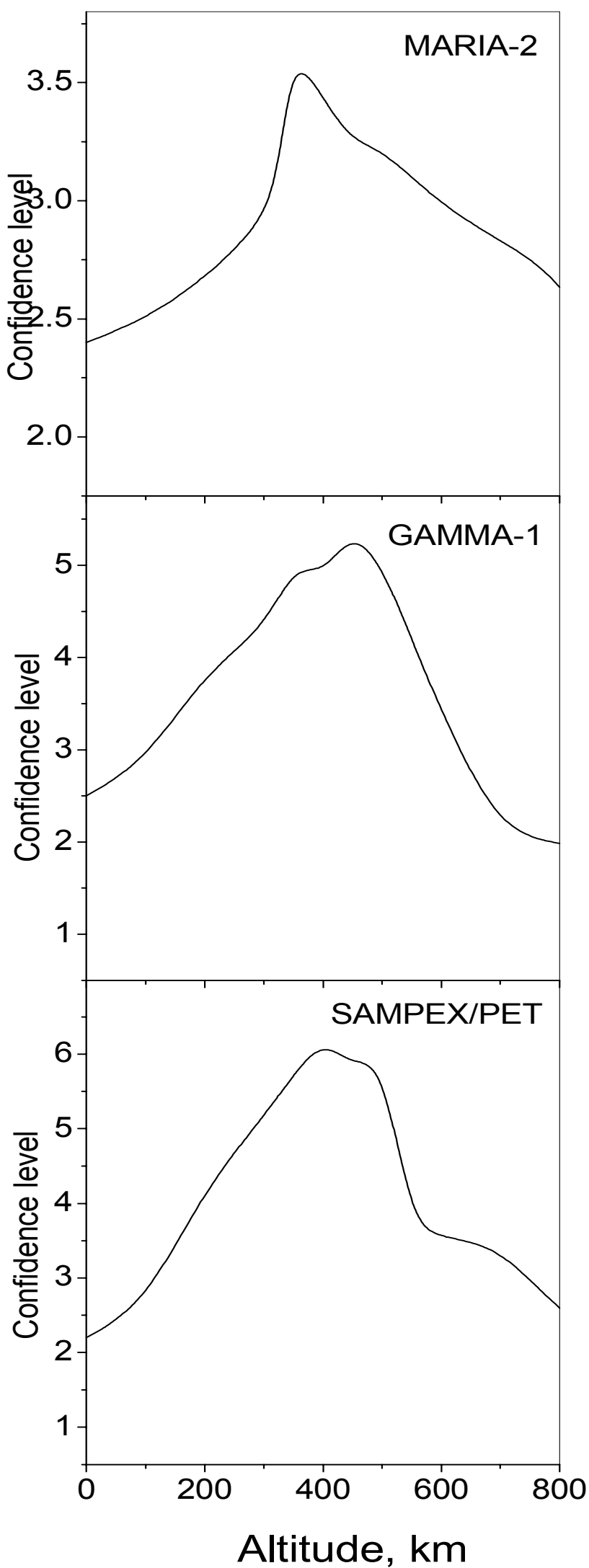

Fig. 5. Confidence level (in number of standard deviations) of the peak in the $\Delta T$ distribution histograms vs. assumed value for the altitude of EME capture in a geomagnetic field tube (MARIA-2, GAMMA-1 and SAMPEX/PET). 
$300-500 \mathrm{~km}$, corresponding to this maximum, practically coincides with the altitude range of the maximum density of ionospheric plasma and agrees with altitudes of EME capture obtained independently in direct satellite experiments on the study of electromagnetic emission of seismic origin (Molchanov et al., 1992).

\section{Conclusion}

New experimental results (MARIA-2, GAMMA-1, ELECTRON and PET instruments) on the observation of highenergy charged particle bursts in near-Earth space give an evidence for the temporal and spatial correlation between particle bursts and earthquakes and are in accordance with the previous work.

The temporal correlation appears as a clear peak in the $\Delta T$ distribution histograms. The positive shift of this peak $(2-5 \mathrm{~h})$ means that the particle bursts can appear as shortterm earthquake precursors. The L-shells of particle bursts and the corresponding earthquake are practically the same.

The altitude of EME capture in the geomagnetic field tube, evaluated in this work from the experimental data on particle bursts, is $300-500 \mathrm{~km}$. This result practically coincides with the data of direct satellite measurements of EME of seismic origin and corresponds to the physical model of seismomagnetosphere correlation considered here.

It is necessary to continue further studies on the physical processes of seismo-magnetospheric correlation. It should be noted that this phenomenon could be useful for the development of earthquake forecasting methods.

Acknowledgements. We acknowledge the Russian Foundation for Basic Research (grant 01-02-16442), which partially supported this work. We acknowledge the Northern California earthquake Data Center (NCEDC), the organizations that contributed the data to the Data Center for creation of CNSS earthquake catalog and the National Space Science Data Center for providing of SAMPEX Level 2 Data.

Topical Editor G. Chanteur thanks R. Spillantini and another referee for their help in evaluating this paper.

\section{References}

Akimov, V. V., Voronov, S. A., Galper, A. M., et al.: Gammaray telescope GAMMA-1, Space Science Review, 49, 111-124, 1988.

Aleshina, M. E., Galper, A. M., Koldashov, S. V., et al.: Interrelation between locations of charged particle precipitation regions and earthquake epicentres, Cosmic Research, 30, 6, 79-81, 1992.
Baker, D. N., Mason, G. M., Figueroa, O., et al.: An Overview of the Solar, Anomalous, and Magnetospheric Particle Explorer (SAMPEX) Mission, IEEE Trans. Geosciences and Remote Sensing, 31, 531-541, 1993.

Fraser-Smith, A. S, Bernard, A., and McGill, P. R.: Low-Frequency Magnetic Field Measurements near the Epicenter of the Ms 7.1 Lomo Prieto earthquake, Geophys. Res. Lett, 17, 9, 1465-1468, 1990.

Galper, A. M., Grachev, V. M., Dmitrenko, V. V., et al.: Study of high-energy electron fluxes on board INTERCOSMOSBULGARIA 1300 satellite, Cosmic Res., 21, 5, 707-709, 1983.

Galper, A. M., Dmitrenko, V. V., Nikitina, N. V., et al.: Interrelation of fluxes of high energy charged particles in radiation belt with seismicity of Earth, Cosmic Res., 27, 5, 789-792, 1989.

Galper, A. M., Koldashov, S. V., and Voronov, S. A.: High-energy particle flux variation as earthquake predictors, Adv. Space Res., 15, 131-133, 1995.

Galper, A. M., Koldashov, S. V., Murashov, A. M., et al.: Highenergy charged particle waves on the low boundary of the radiation belt, Proc. of 25th ICRC, 2, 349-350, 1997.

Galper, A. M., Koldashov, S. V., and Murashov, A. M.: Numerical modeling of physical processes on the atmospheric boundary of radiation belt, Cosmic Res., 38, 1, 70-79, 2000.

Galperin, Yu. I., Gladyshev, V. A., Jordjio, N. V., et al.: Precipitation of high-energy captured particles in the magnetosphere above epicenter of an incipient earthquake, Cosmic Res., 30, 1, 89-106, 1992.

Malone, S., Gomberg, J., Ludwin, R., et al.: The Council of the National Seismic System and a composite earthquake catalog for the United States, IRIS Newsletter, XV, 1, 6-9, 1996.

Molchanov, O. A., Majaeva, O. A., and Protopopov, M. L: Observation of electromagnetic emissions of seismic origin on board INTERCOSMOS-24 satellite, Cosmic Res., 32, 6, 128-137, 1992.

Molchanov, O. A. and Majaeva, O. A.: Statistic features of ULF and VLF electromagnetic emissions in upper ionosphere and magnetosphere above centers of earthquakes, Cosmic Res., 32, 4-5, 150-157, 1994.

Pustovetov, V. P. and Malyshev, A. V.: Spatial-temporal correlation of the earthquakes and variations of high-energy particle flux in the inner radiation belt, Cosmic Res., 31, 5, 84-87, 1993.

Voronov, S. A., Galper, A. M., Koldashov, S. V., et al.: Registration of Sporadic Increase of High Energy Particle Flux near Brazilian Anomaly Region, Proc. of 20th ICRC, 4, 451-452, 1987.

Voronov, S. A., Galper, A. M., Koldashov, S. V., et al.: Observation of high-energy charged particle flux increases in SAA region in 10 September 1985, Cosmic Res., 27, 4, 629-631, 1989.

Voronov, S. A., Galper, A. M., Koldashov, S. V., et al.: Increase of high-energy charged particle fluxes in SAA region and the Earth's seismic activity, Cosmic Res., 28, 5, 789-791, 1990.

Voronov, S. A., Galper, A. M., Koldashov, S. V., et al.: MARIA2 charged particles' magnetic spectrometer, Pribori i Tehnika Eksperimenta, 2, 59-63, 1991. 